\section{Matrix metalloproteinase 9 as a marker of disease activity in multiple sclerosis}

Matrix metalloproteinase 9 (MMP9) levels in serum and cerebrospinal fluid (CSF) are known to be elevated in patients with multiple sclerosis; these patients also have lower than normal levels of tissue inhibitor of metalloproteinase 1 (TIMP1), an inhibitor of MMP9. The MMP9:TIMP1 ratio has been proposed as a biomarker of multiple sclerosis activity. Fainardi et al. measured MMP9 activity and TIMP1 levels in 61 consecutive patients with multiple sclerosis; patients with other neurological disorders acted as controls.

Mean CSF levels of active MMP9, CSF MMP9: TIMP1 ratios, and intrathecal MMP9 synthesis were substantially higher in patients with multiple sclerosis than in those with noninflammatory neurological disorders. The serum MMP9: TIMP1 ratio was higher in patients with multiple sclerosis and in those with other inflammatory disorders than in patients with noninflammatory disorders. Patients with clinical and MRI evidence of active disease had higher serum levels of active MMP9, higher serum MMP9: TIMP1 ratios and higher intrathecal production of active MMP9 than patients with stable disease, but CSF levels of active MMP9 and CSF active MMP9:TIMP1 ratios were elevated only in patients with gadolinium-enhancing lesions on MRI.

The results suggest that high CSF levels of active MMP9 are selectively associated with multiple sclerosis, while high serum MMP9 is also found in other inflammatory neurological disorders. CSF and serum ratios of active MMP9 to TIMP1 are an important indicator of disease activity, with high CSF levels of active MMP9 associated with more-severe disease.

Original article Fainardi E et al. (2006) Cerebrospinal fluid and serum levels and intrathecal production of active matrix metalloproteinase-9 (MMP-9) as markers of disease activity in patients with multiple sclerosis. Mult Scler 12: 294-301

\section{Dystrophin expression in muscle after injection of donor myogenic cells}

Researchers in Canada have evaluated the effect of the implantation of donor muscle precursor cells (MPCs) on the expression of dystrophin in patients with Duchenne muscular dystrophy. In nine patients, cultured MPCs from a parent were injected at $1-2 \mathrm{~mm}$ intervals into a $0.25 \mathrm{~cm}^{3}$ or $1 \mathrm{~cm}^{3}$ section of a tibialis anterior muscle. Control injections of saline were administered to the contralateral muscle of each patient.

Muscle biopsies taken 4 weeks after MPC implantation revealed expression of donor dystrophin in eight patients, with the percentage of myofibers expressing dystrophin varying from $3.5 \%$ to $26 \%$. In addition to hybrid myofibers resulting from fusion of MPCs with existing fibers, small sections of entirely dystrophinpositive fibers, resulting from fusion of implanted MPCs with one another, were detected.

Previous attempts to induce dystrophin expression by MPC implantation in patients with muscular dystrophy have produced disappointing results. The authors state that a high density of injections is necessary for successful implantation, as MPCs do not diffuse from the injection site but fuse with fibers reached by the injection; fusion with fibers might also be assisted by damage caused by the injections. Previous studies used immunosuppressive agents such as ciclosporin or cyclophosphamide, which can damage implanted cells; the present study used tacrolimus, which seemed effective in preventing graft rejection in most cases, although substantial lymphocyte infiltration of the graft site was observed in two patients. Monitoring the immune response by comparison of graft and control sites is important, say the authors.

Original article Skuk D et al. (2006) Dystrophin expression in muscles of Duchenne muscular dystrophy patients after high-density injections of normal myogenic cells.

J Neuropathol Exp Neurol 65: 371-386

\section{Electrophysiological parameters might be useful markers of HD progression}

Huntington's disease (HD) affects a wide variety of motor, cognitive and behavioral functions. Patients generally deteriorate over time, but as disease course varies widely it has been difficult to establish scales for longitudinal followup studies. Currently available scales such as the Unified Huntington's Disease Rating Scale (UHDRS) are time-consuming to use and partly subjective. Electrophysiological alterations have been described in HD, however, and 